\title{
Geometrical structure of an iron epilayer on Si (111) : an X-ray standing wave analysis
}

\author{
J. C. Boulliard, B. Capelle, \\ D. Ferret, \\ A. Lifchitz, \\ C. Malgrange, \\ J. F. Pétroff, \\ A. Taccoen and Y. L. Zheng \\ Laboratoire de Minéralogie et Cristallographie, Universités Paris VI et Paris VII $\left(^{*}\right), 4$ place \\ Jussieu, 75252 Paris Cedex 05, France
}

(Received 11 February 1992, accepted in final form 6 March 1992)

\begin{abstract}
The structure of an iron film, deposited at low temperature $\left(50^{\circ} \mathrm{C}\right)$ upon a silicon (111) substrate, has been determined by means of X-ray Standing Wave experiments performed at LURE (Orsay, France). Experimental results are coherent with the model of an abrupt interface between the adsorbate and the surface : the first site of adsorption terminates the bulk silicon and a body-centred iron layer epitaxially grows on the substrate with a preferential growth orientation.
\end{abstract}

\section{Introduction.}

For years, the interfaces between a semiconductor or a metal and a silicon crystal have received much attention due to their potential technological applications. Among all the possible heterostructures, the silicide-silicon systems are of special interest; some of them display a quasi-perfect interface and they may allow silicon to be the source of many new electronic and optoelectronic devices. Among the semiconducting disilicides, the $\beta$-FeSi ${ }_{2}$ with a direct gap of $0.85 \mathrm{eV}[1,2]$ appears as one promising material. Different authors have studied its epitaxy on Si (111) and Si (100) using solid phase epitaxy (SPE) [3, 4]. Since the first stage of this kind of growth is the deposition of an iron film on the substrate, a better knowledge of the $\mathrm{Fe} / \mathrm{Si}$ (111) system is a prerequisite. If a model exists for the structure of the iron film [5], the quality of the interface and the position of the first site of absorption are not clearly established.

Studies about other transition metals show various behaviours : silver on Si (111) presents an abrupt interface [6], while cobalt and silicon seem to interdiffuse [7]. First results of X-ray standing wave experiments (XSW) concerning the Fe/Si (111) are presented here. This method allows us to precisely determine the location of the deposited atoms along the surface and is complementary to other structural techniques such as I-V LEED and SEXAFS (Surface Extended X-ray Absorption Fine Structure).

$\left(^{*}\right)$ CNRS URA9. 


\section{Theory.}

An XSW experiment usually consists in simultaneously recording the rocking curve of the substrate and the fluorescence yield of adsorbate atoms upon the surface. When a crystal is at a Bragg diffraction position, interferences occur between the incident and the diffracted waves leading to periodic standing waves with nodal and antinodal planes parallel to the diffraction planes and having the same periodicity. According to $\mathrm{X}$-ray dynamical theory [810], the nodes move by half a period when rotating the crystal through the rocking curve. Then the fluorescence yield of adsorbate atoms is very sensitive to the atom position when rocking the crystal through a Bragg reflection [9, 11, 12]. This method allows us to precisely determine the position of adatoms with respect to the bulk substrate lattice. When the diffraction planes are parallel to the surface of the crystal, XSW experiments give the height of adsorbed atoms above the surface. Using other reflections such as 220 for a Si (111) crystal, one may determine the position of adatoms by means of a geometrical triangulation.

Outside the crystal, the intensity of the X-ray standing wave $|D(\mathbf{r}, \theta)|^{2}$ is :

$$
\begin{aligned}
|D(\mathbf{r}, \theta)|^{2}=\left|D_{0}+D_{\mathrm{h}}(\theta) \mathrm{e}^{-i 2 \pi \mathbf{h} \cdot \mathbf{r}}\right|^{2} & = \\
& =\left|D_{0}\right|^{2}\left\{1+|\xi(\theta)|^{2}+2|\xi(\theta)| \cos (\Psi(\theta)-2 \pi \mathbf{h} \cdot \mathbf{r})\right\}
\end{aligned}
$$

where $D_{0}$ and $D_{h}(\theta)$ are the incident and reflected waves, respectively, $|\xi(\theta)|^{2}=$ $\frac{\left|D_{\mathrm{h}}(\theta)\right|^{2}}{\left|D_{0}\right|^{2}}$ is the $\mathrm{X}$-ray reflectivity, $\Psi(\theta)$ the phase of the reflected wave and $h$ the diffraction vector. The normalized fluorescence yield, $Y(\theta)$, is proportional to the intensity of the $\mathrm{X}$-ray standing wave $|D(\mathbf{r}, \theta)|^{2}$ and the core electron distribution $\rho(\mathbf{r})$ :

$Y(\theta) \propto \frac{\int \rho(\mathbf{r})|D(\mathbf{r}, \theta)|^{2} \mathrm{~d}^{3} \mathbf{r}}{\int \rho(\mathbf{r}) \mathrm{d}^{3} \mathbf{r}} \propto 1+|\xi(\theta)|^{2}+2|\xi(\theta)| \frac{\int \rho(\mathbf{r}) \cos (\Psi(\theta)-2 \pi \mathbf{h} \cdot \mathbf{r}) \mathrm{d}^{3} \mathbf{r}}{\int \rho(\mathbf{r}) \mathrm{d}^{3} \mathbf{r}}$

where $\rho(\mathbf{r})$, to an excellent approximation, reduces to the atomic distribution. If one defines the Fourier component, with respect to the diffraction vector $\mathbf{h}$, of $\rho(\mathbf{r})$ by its amplitude $F$ and phase $P$ as follows :

$$
F \mathrm{e}^{i 2 \pi P}=\frac{\int \rho(\mathbf{r}) \mathrm{e}^{i 2 \pi \mathbf{h} \cdot \mathbf{r}} \mathrm{d}^{3} \mathbf{r}}{\int \rho(\mathbf{r}) \mathrm{d}^{3} \mathbf{r}}
$$

then, the experimental fluorescence yield $Y(\theta)$ directly provides the values of $F$ and $P$ :

$$
Y(\theta)=1+|\xi(\theta)|^{2}+2|\xi(\theta)| F \cos (\Psi(\theta)-2 \pi P)
$$

The XSW analysis consists in relating the experimentally determined $F$ and $P$ parameters to the structure of the epilayer $\rho(\mathbf{r})$. The relationship between these parameters and $\rho(\mathbf{r})$ is developed below for some usual cases and for symmetric reflections. Symmetric 
reflections occur when the projection of the normal $\mathbf{n}$ to the crystal surface on the plane formed by the incident and diffracted beams is parallel to the diffraction vector $\mathbf{h}$. Two cases can be distinguished :

i) parallel symmetric case, which is the usual symmetric case where $\mathbf{n}$ and $\mathbf{h}$ are parallel ;

ii) inclined symmetric case, when $\mathbf{n}$ and $\mathbf{h}$ are not parallel.

Let us first consider a parallel symmetric reflection and call $z$ the coordinate along the normal $\mathbf{n}$. Then only the projected atomic distribution $\rho(z)$ on the normal $\mathbf{n}$ will be involved in the calculation of the $F$ and $P$ parameters :

$$
F \mathrm{e}^{l 2 \pi P}=\frac{\int \rho(\mathbf{r}) \mathrm{e}^{i 2 \pi \mathbf{h} \cdot \mathbf{r}} \mathrm{d}^{3} \mathbf{r}}{\int \rho(\mathbf{r}) \mathrm{d}^{3} \mathbf{r}}=\frac{\int_{0}^{t} \rho(z) \mathrm{e}^{i 2 \pi z / d_{h k l}} \mathrm{~d} z}{\int_{0}^{t} \rho(z) \mathrm{d} z}
$$

where $d_{h k l}=1 /|\mathbf{h}|$ and $t$ is the thickness of the layer.

The meaning of the parameters $F$ and $P$ can be understood in the particular case of one monolayer of atoms. One can define such a distribution as :

$$
\rho(z)=\rho^{0}(z) \otimes \delta\left(z-d_{\mathrm{IF}}\right) \quad \text { with } \otimes: \text { convolution }
$$

where $d_{\mathrm{IF}}$, called the interface distance (Fig. 1), is the average distance between the atoms of the monolayer and the nearest downward diffraction plane of the substrate. $\rho^{0}(z)$ is the
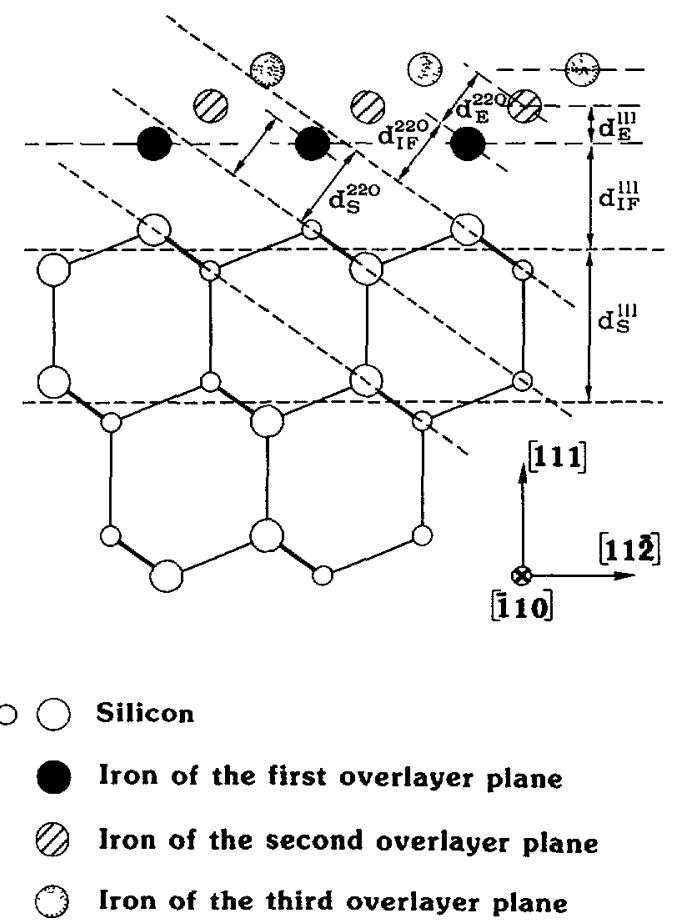

Fig. 1. - Schematic illustration of a silicon (111) surface, covered with an ordered 3 ML film : side view along [110]. The diffraction planes are drawn as dashed lines. 
projected atomic distribution around this average distance $d_{\mathrm{IF}}$ and it is here normalized to unity for convenience. In this case, $F$ and $P$ can be written as :

$$
F \mathrm{e}^{i 2 \pi P}=f_{\mathrm{c}} \mathrm{e}^{i 2 \pi d_{\mathrm{IF}} / d_{h k \ell}} \quad \text { with } \quad f_{\mathrm{c}}=\int \rho^{0}(z) \mathrm{e}^{i 2 \pi z / d_{h k l}} \mathrm{~d} z
$$

Thus from the phase parameter $P=d_{\mathrm{IF}} / d_{h k l}$, the interface distance $d_{\mathrm{IF}}$ is obtained. The parameter $F=f_{c}$ describes the order of the layer : for a completely ordered monolayer $\left(\rho^{0}(z)=\delta(z)\right), F=f_{\mathrm{c}}=1$ and for a completely unordered monolayer, $F=f_{\mathrm{c}}=0$. For this reason, $f_{\mathrm{c}}$ is called the coherent fraction.

The case with $N$ identical monolayers [13,14] can be easily described by

$$
\rho(z)=\rho^{0}(z) \otimes \sum_{k=0}^{N-1} \delta\left(z-d_{\mathrm{IF}}-k d_{\mathrm{E}}\right)
$$

where $d_{\mathrm{E}}$ is the distance between the layers (Fig. 1). $F$ and $P$ are then related to the coherent fraction of a monolayer $f_{\mathrm{c}}$ and the interface distance $d_{\mathrm{IF}}$ by

$$
F \mathrm{e}^{i 2 \pi P}=f_{\mathrm{c}} \frac{\sin \left(N \pi d_{\mathrm{E}} / d_{h k \ell}\right)}{N \sin \left(\pi d_{\mathrm{E}} / d_{h k \ell}\right)} \mathrm{e}^{i 2 \pi\left\{d_{\mathrm{IF}} / d_{h k \ell}+\frac{N-1}{2} d_{\mathrm{E}} / d_{h k \ell}\right\}}
$$

For the case of inclined symmetric reflections the diffraction vector $\mathbf{h}$ is not parallel to the normal $\mathbf{n}$ to the surface. Nevertheless, it can be treated similarly to the case of parallel symmetric reflections by using the projected atomic distribution along the vector $h$. Let $\tau$ be the coordinate along $h$ and $\rho_{\mathrm{h}}(\tau)$ be the projected distribution (Appendix I). In general, $\rho_{\mathrm{h}}(\tau)$ is defined for $\tau$ varying from $-\infty$ to $+\infty$. The parameters $F$ and $P$ are deduced from $\rho_{\mathrm{h}}(\tau)$ by

$$
F \mathrm{e}^{i 2 \pi P}=\frac{\int \rho(\mathbf{r}) \mathrm{e}^{i 2 \pi \mathrm{h} \cdot \mathbf{r}} \mathrm{d}^{3} \mathbf{r}}{\int \rho(\mathbf{r}) \mathrm{d}^{3} \mathbf{r}}=\lim _{L \rightarrow+\infty} \frac{\int_{-L}^{L} \rho_{\mathrm{h}}(\tau) \mathrm{e}^{i 2 \pi \tau / d_{h k \mathrm{l}}} \mathrm{d} \tau}{\int_{-L}^{L} \rho_{\mathrm{h}}(\tau) \mathrm{d} \tau}
$$

Two cases may happen depending on the nature of the interface :

i) if the lattices of the epilayer and substrate do not match at the interface, $\rho_{\mathrm{h}}(\tau)$ does not have a period equal to a multiple of $d_{h k \ell}$ and $F \mathrm{e}^{i 2 \pi P}=0$. The inclined symmetric reflection does not provide any information on the layer;

ii) if the lattice matching occurs (epitaxial case), $\rho_{\mathrm{h}}(\tau)$ has a period equal to a multiple of $d_{h k \ell}$ (Appendix I) and the parameters $F$ and $P$ are given by :

$$
F \mathrm{e}^{i 2 \pi P}=\frac{\int_{\text {over } \tau_{\mathrm{h}}} \rho_{\mathrm{h}}(\tau) \mathrm{e}^{i 2 \pi \tau / d_{h k \ell}} \mathrm{d} \tau}{\int_{\text {over } \tau_{\mathrm{h}}} \rho_{\mathrm{h}}(\tau) \mathrm{d} \tau}
$$

where $\tau_{\mathrm{h}}=n d_{h k \ell}$ ( $n$ integer) represents the period of $\rho_{\mathrm{h}}(\tau)$ and the integration is performed over one period $\tau_{\mathrm{h}}$. 
This means that inclined symmetric reflections indicate whether the lattice of the epilayer matches the substrate lattice at the interface. From the previous formula and similarly to the case of parallel symmetric reflections, it can be easily established that :

i) for the case of one atomic site over one period $\tau_{h}$.

$$
\begin{aligned}
\rho_{\mathrm{h}}(\tau) & =\rho_{\mathrm{h}}^{0}(\tau) \otimes \delta\left(\tau-d_{\mathrm{IF}}\right) \otimes \sum_{m=-\infty}^{+\infty} \delta\left(\tau-m \tau_{\mathrm{h}}\right) \\
F \mathrm{e}^{i 2 \pi P} & =f_{\mathrm{c}} \mathrm{e}^{i 2 \pi d_{\mathrm{IF}} / d_{h k l}} \quad \text { with } \quad f_{\mathrm{c}}=\int_{\text {over } \tau_{\mathrm{h}}} \rho_{\mathrm{h}}^{0}(\tau) \mathrm{e}^{i 2 \pi \tau / d_{h k l}} \mathrm{~d} \tau
\end{aligned}
$$

where $d_{\mathrm{IF}}$ is the mean distance between the atoms and the nearest diffraction plane and $\rho_{\mathrm{h}}^{0}(\tau)$ is the atomic distribution around this mean value $d_{\mathrm{IF}}$;

ii) for the case of $N$ equally spaced atomic sites over one period $\tau_{\mathrm{h}}$.

$$
\begin{aligned}
\rho_{\mathrm{h}}(\tau) & =\rho_{\mathrm{h}}^{0}(\tau) \otimes \sum_{k=0}^{N-1} \delta\left(\tau-d_{\mathrm{IF}}-k d_{\mathrm{E}}\right) \otimes \sum_{m=-\infty}^{+\infty} \delta\left(\tau-m \tau_{\mathrm{h}}\right) \\
F \mathrm{e}^{i 2 \pi P} & =f_{\mathrm{c}} \frac{\sin \left(N \pi d_{\mathrm{E}} / d_{h k \ell}\right)}{N \sin \left(\pi d_{\mathrm{E}} / d_{h k \ell}\right)} \mathrm{e}^{i 2 \pi\left\{d_{\mathrm{FF}} / d_{h \ell \ell}+\frac{N-1}{2} d_{\mathrm{E}} / d_{h k \ell}\right\}}
\end{aligned}
$$

where $d_{\mathrm{E}}$ is the spacing between the projections along $\mathbf{h}$ of the different atomic sites.

It can be concluded that the same treatment can be used to determine the structural parameters $f_{\mathrm{c}}$ and $d_{\mathrm{IF}}$ from the experimental values of $F$ and $P$, for both parallel and inclined symmetric reflections. The positions of the first atoms of the epilayer are then deduced by geometric reconstruction from the $d_{\mathrm{IF}}$ values determined for different reflections. Inclined symmetric reflections are particularly useful, since they provide information on the quality of the matching of both lattices at the interface.

\section{Experimental arrangement.}

3.1 TWO-AXIS SPECTROMETER. - The experimental arrangement $[15,16]$, installed on beam line D15B-DCI, is a double-crystal spectrometer with horizontal axes to preserve the polarization properties of the synchrotron radiation. This spectrometer consists of two goniometric holders and two slit collimators (each one being placed upstream a goniometer). It is fixed on a heavy marble stand. The rotations are driven by stepping motors and are controlled by means of precise optical devices. The entire set-up is controlled through a microcomputer and an integrated software, especially designed for XSW tasks.

The first crystal is the $\mathrm{X}$-ray monochromator and the second one is the sample. When rotating the crystal around the Bragg position, the reflectivity is recorded by means of a scintillation counter ( $\mathrm{NaI}$ crystal), and sent to the computer where feedback corrections are calculated via a numerical servo-loop. Fluorescence from the sample is detected by means of a solid state $\mathrm{Si}(\mathrm{Li})$ detector mounted on a support which allows angular adjustments and vertical and horizontal translations. A multichannel analyzer (MCA) card is used to store fluorescence spectra in the microcomputer. The incident photon flux after the monochromator is measured through an ionization chamber in order to normalize both signals (the reflectivity and the fluorescence).

Precise angular positioning and its measurement are among the main difficulties of this type of setting. As the fluorescence signal can exhibit significant variations in an angular range of three or four times the width of the rocking curve, a rotation range of typically 10 to 
30 arc seconds and a rotation accuracy of several hundredths of arc second are required. An electronic feedback loop achieves a precise positioning by means of heavy lever arms, a piezoelectric device and a differential capacitive sensor. The advantage is that there is no heat production and no mechanical resonance by preloading the lever arm. The dynamic angular position is stable with a precision of $0.01^{\prime \prime}$.

In order to increase the photon fluorescence yield, the incident monochromatized beam is adjusted at a wavelength as close as possible to the absorption $\mathbf{K}$-edge of the analysed element. Moreover it is necessary to reduce the diffused intensity (mainly the Compton scattering) which partially overlaps the fluorescence signal. This diffuse intensity varies proportionally to $\left(\mathbf{e}_{1} \cdot \mathbf{e}_{2}\right)^{2}, \mathbf{e}_{1}$ and $\mathbf{e}_{2}$ being the polarization vectors of the incident and diffused photons respectively [17]. The beam being mainly polarized in the horizontal plane, the solid state detector is oriented perpendicularly to the incident beam, as close as possible to the horizontal polarization direction.

The set-up is housed in a lead-covered room which avoids any X-ray leakage as well as rapid thermal variations. The thermal drift without any correction is close to one arc second per hour when the stationary thermal state is reached, i.e. after one day. The high brilliancy $\mathrm{X}$-ray beam provided by the storage ring allows fluorescence signals which are intense enough to perform experiments in a few hours. During this time, this XSW setting exhibits a good mechanical stability and samples are rotated with a static precision better than $0.01^{\text {" by means }}$ of two feedback loops : the first one, analogical, ensures the linearity of the piezoelectric transducer and the second one, which is numerical, ensures the correction of the long term thermal drift. The computerized control and operating systems allow a high flexibility.

3.2 Monochromators. - To record significant data the $X$-ray beam incident on the sample should be as close as possible to a plane wave. Therefore, a monochromator should deliver a beam with properties which may be listed as follows :

i) an angular divergence $\delta$ much smaller than the intrinsic reflection width of the sample ;

ii) a narrow spectral window when a non-dispersive setting is not used;

iii) a low harmonics contamination;

iv) tunability.

For this study original monolithic grooved four-reflection monochromators have been developed (Fig. 2). This design allows us to work in the $(+,-)$ parallel setting. In areas I and II, the diffraction planes are parallel to the surface. The double symmetric reflection is efficient in reducing the tails of the reflection curve $[18,19]$. In area III, the diffraction planes make an angle $\alpha$ with the crystal surface. Asymmetry is characterized by the factor

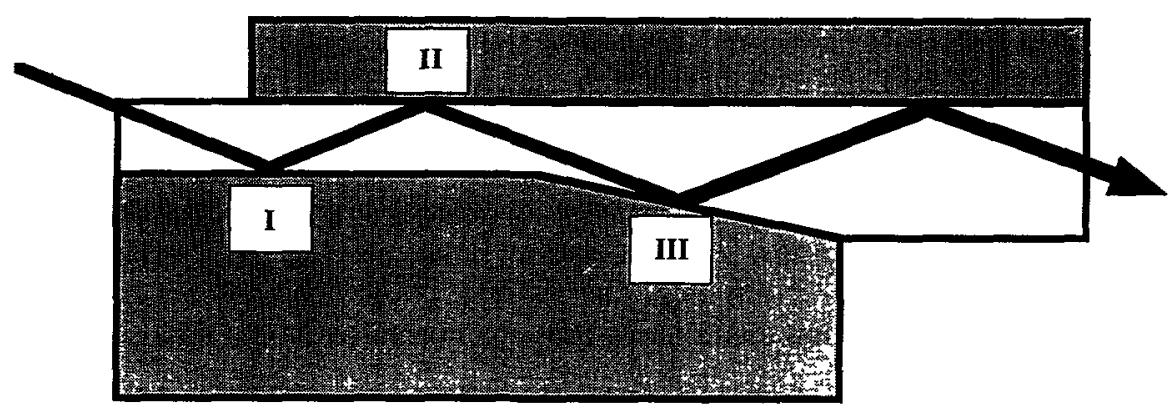

Fig. 2. - Schematic drawing of a monolithic four reflections monochromator. The bold line indicates the X-ray beam. 
$b$ defined as :

$$
b=\frac{\sin \left(\theta_{\mathrm{B}}-\alpha\right)}{\sin \left(\theta_{\mathrm{B}}+\alpha\right)}
$$

where $\theta_{\mathrm{B}}$ is the Bragg angle.

Let $\omega_{\mathrm{s}}$ be the intrinsic width of a Bragg symmetric reflection. The angular range accepted by area III is, to a good approximation, $\omega_{s} / \sqrt{b}$ and the reflected one $\omega_{\mathrm{s}} \sqrt{b}$. The interest of the asymmetric reflection lies not only in the reduction of the angular divergence of the exit beam [20], but also in the rejection of the harmonics by means of a refraction effect [21]. The reflection curve of area II overlaps with the acceptance curve of area III for the fundamental wavelength and the overlapping is very narrow for the harmonics. Finally a fourth symmetric reflection is used to obtain an exit beam parallel to the incident one.

Two silicon monochromators have been designed and built, one for the 111 reflections, the other one for 220 .

These monochromators are tunable, but, in fact, the choice of the wavelength $\lambda$ to be used is a compromise. Several parameters have to be taken into account : i) the ratio of the angular divergence $\delta$ over $\omega_{\mathrm{s}}$, ii) the wavelength distribution of the synchrotron radiation, iii) the absorption edge of the excited atom (1.74 $\AA$ for FeK), iv) the decrease of the fluorescence yield for wavelengths smaller than the absorption edge and $v$ ) the presence of the nearest harmonic ( $\lambda / 2$ for the 220 reflection, $\lambda / 3$ for the 111 reflection). In view of all these considerations, we chose a smaller wavelength for the 220 reflection than for the 111 reflection :

i) $\lambda \approx 1.6 \AA$ for the 111 monochromator where $\alpha=7^{\circ}$,

ii) $\lambda \approx 1.2 \AA$ for the 220 one where $\alpha=10^{\circ}$.

Figure 3 shows, for the 220 reflection, the expected rocking curve of the sample which is very similar to the intrinsic one. The contamination of the beam by harmonics has been measured and the results are very good : $0.1 \%$ for the 111 monochromator and $0.5 \%$ for the 220 one.

The characteristics of the monochromators used in the present work are listed in table I.

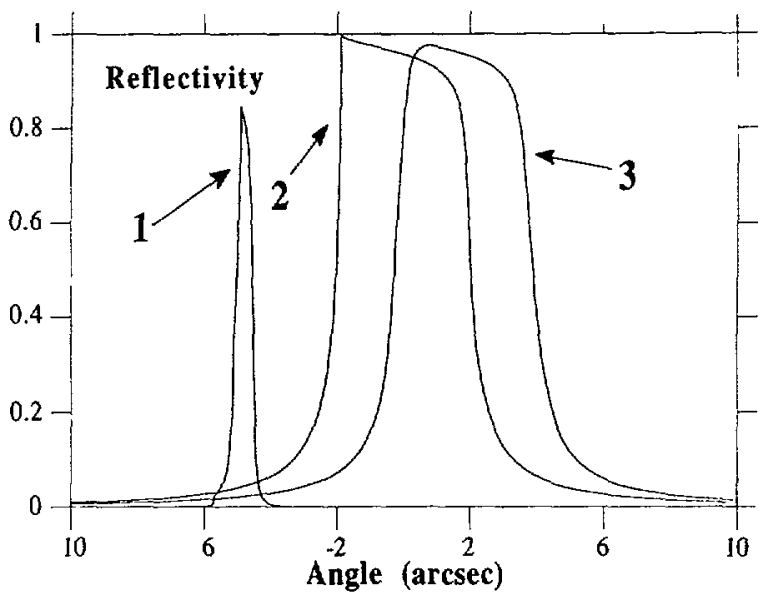

Fig. 3. - Calculated reflectivities (220 reflection, $\lambda=1.2 \AA$ ): (1) for the monochromator, (2) for the sample if an incident plane wave is assumed and (3) for the sample illuminated by the monochromator beam. The positions of the profiles, along the horizontal axis, are arbitrary. 
Table I.

\begin{tabular}{|c|c|c|}
\hline Monochromator & 111 & 220 \\
\hline Asymmetry angle $\alpha$ & $7^{\circ}$ & $10^{\circ}$ \\
\hline Tunability & $1.3-2.6 \AA$ & $0.9-1.9 \AA$ \\
\hline Wavelength used & $1.56 \AA$ & $1.19 \AA$ \\
\hline$\omega_{\mathrm{s}}^{1}$ & $7.68^{\prime \prime}$ & $4.05^{\prime \prime}$ \\
\hline$\omega_{s}^{\mathrm{e}}$ & $7.93^{\prime \prime}$ & $4.20^{\prime \prime}$ \\
\hline
\end{tabular}

$\omega_{\mathrm{s}}^{1}$ : full width at half maximum of the intrinsic profile; $\omega_{\mathrm{s}}^{\mathrm{e}}$ : full width at half maximum of the profile, when the sample is illuminated by the monochromator beam. $\omega_{\mathrm{s}}^{1}$ and $\omega_{\mathrm{s}}^{\mathrm{e}}$ are calculated for the wavelengths used.

3.3 SAMPLE FEATURES. - Substrates are (111) n-doped silicon wafers with a diameter of $50 \mathrm{~mm}$ and a thickness of $2 \mathrm{~mm}$. This unusual thickness for a wafer has been chosen to minimize the strains due to the heat treatments. Indeed, the quality of the substrate is crucial for XSW measurements. Their crystalline quality is checked by X-ray topography. Deposition of iron on silicon is made at the Centre de recherches sur les Mécanismes de Croissance Cristalline at Marseille (France). First, the substrate is cleaned ex situ with a dilute hydrofluoric acid etching and rinsed in pure water. The substrate is cleaned in the Molecular Beam Epitaxy machine by heating it at $800^{\circ} \mathrm{C}$ and by means of an atomic silicon beam on the surface. Then the state of the surface is controlled by RHEED and the final pattern exhibits a sharp $7 \times 7$ reconstruction. During the deposition of iron on the silicon wafer, the pressure in the UHV chamber of the MBE machine varies between $2 \times 10^{-10}$ and $10^{-9}$ torr. The substrate temperature is $50^{\circ} \mathrm{C}$, the temperature of the iron source is $1200^{\circ} \mathrm{C}$ and the time of deposition varies from a few seconds to a few minutes.

For the sample used in this study the iron deposition time has been $1 \mathrm{mn}$. Since at the present time the XSW set-up is operating at air, the iron film was protected by a $15 \AA$ thick amorphous silicon layer deposited in situ with a source at $1460^{\circ} \mathrm{C}$. The amount of iron on the silicon wafer has been measured afterwards by Rutherford Back Scattering (RBS) and $2.35 \times 10^{15}$ atoms per $\mathrm{cm}^{2}$ were detected.

\section{Results.}

Several experiments were performed, using 111 and 220 reflections. Since iron is present in many parts of the experimental arrangement, the experiment must be protected from any parasitic fluorescence. A reference silicon substrate is first analysed to check that no iron fluorescence appears.

The collection of data is the sum of several scans, each consisting in rocking the crystal through its Bragg position step by step. The duration of one step and their number are two parameters of the measurement. At each step, the reflected intensity is registered by a scintillation counter and the fluorescence emission by a $\mathrm{Si}(\mathrm{Li})$ detector. The recorded fluorescence spectrum consists of three areas : the first one is the $K \alpha$ iron emission peak, the two others, located on each side of the peak, allow a determination of the background which is then substracted. 
Experimental conditions are :

i) for the 111 data : the wavelength $\lambda$ is $1.56 \AA$ and the duration of the experiment four hours. Far from the Bragg position, the iron $\mathrm{K} \alpha$ fluorescence yield is 17 counts per second ;

ii) for the 220 data : the wavelength $\lambda$ is $1.19 \AA$ and the duration of the experiment nine hours. Far from the Bragg position, the iron $K \alpha$ fluorescence yield is 10 counts per second.

Experimental results are reported in figure 4. The experimental curves are fitted with

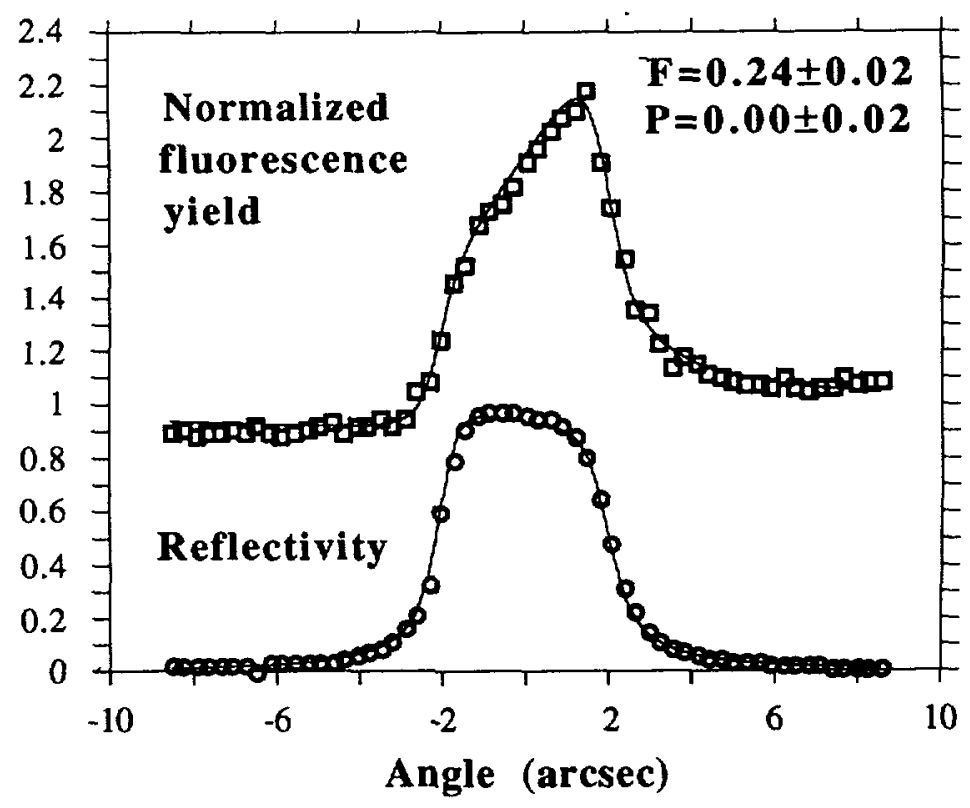

a)

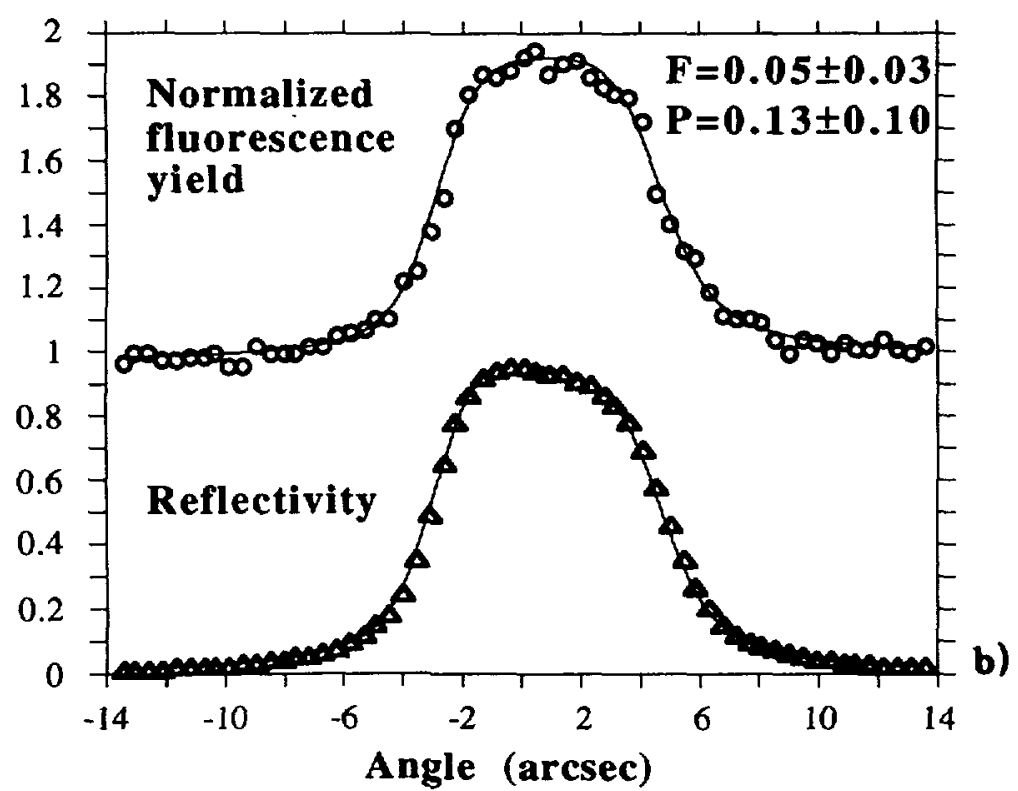

Fig. 4. - Experimental points and theoretical fitted curves for reflectivity and Fe fluorescence yield. a) 220 reflection, $\lambda=1.19 \AA$. b) 111 reflection, $\lambda=1.56 \AA$. 
theoretical curves to extract the $F$ and $P$ parameters (Tab. II). The 111 fluorescence yield is similar to the fluorescence of a totally unordered iron film, but the 220 yield clearly indicates a partially ordered film : it means that, even for a deposition made at a low temperature $\left(50^{\circ} \mathrm{C}\right)$ and for a buried interface, order may be observed.

Table II. $-F$ and $P$ parameters extracted from the XSW data; $f_{\mathrm{c}}$ and $d_{\mathrm{IF}} / d_{h k \ell}$ values for $a$ $\mathrm{ABC}$ stacking.

\begin{tabular}{|c|c|c|}
\hline Reflection & 111 & 220 \\
\hline$F$ & $0.05 \pm 0.03$ & $0.24 \pm 0.02$ \\
\hline$P$ & $0.13 \pm 0.10$ & $0.00 \pm 0.02$ \\
\hline$f_{\mathrm{c}}$ & $0.24 \pm 0.14$ & $0.24 \pm 0.02$ \\
\hline$d_{\mathrm{IF}} / d_{h k l}$ & $0.85 \pm 0.10$ & $0.96 \pm 0.02$ \\
\hline
\end{tabular}

\section{Structural models and discussion.}

5.1 PSEUDOMORPHIC GROWTH MODEL. - A pseudomorphic growth of Fe films, deposited at room temperature on $\mathrm{Si}$ (111), was proposed for the first time by Kanaji et al. [5] from I-V LEED observations. The $\mathrm{Fe}$ epilayer has the $\alpha \mathrm{Fe}$ bulk structure and the relationships between epilayer and substrate are :

$$
\mathrm{Fe}(111) / / \mathrm{Si}(111) \quad \text { and } \mathrm{Fe}[\overline{1} 10] / / \mathrm{Si}[\overline{1} 10]
$$

Other publications [22-24] are in agreement with this model.

This epitaxial growth is more easily described by using an hexagonal cell for $\alpha \mathrm{Fe}$ and for $\mathrm{Si}$. The lattice parameters of the hexagonal cells deduced from those of the cubic ones are:

$$
\begin{array}{lll}
\mathbf{a}_{\mathrm{Fe}}^{\prime}=\mathbf{a}_{\mathrm{Fe}}-\mathbf{c}_{\mathrm{Fe}}, & \mathbf{b}_{\mathrm{Fe}}^{\prime}=\mathbf{b}_{\mathrm{Fe}}-\mathbf{a}_{\mathrm{Fe}}, & \mathbf{c}_{\mathrm{Fe}}^{\prime}=\mathbf{a}_{\mathrm{Fe}}+\mathbf{b}_{\mathrm{Fe}}+\mathbf{c}_{\mathrm{Fe}} \\
\mathbf{a}_{\mathrm{S} 1}^{\prime}=1 / 2\left(\mathbf{a}_{\mathrm{Si}_{1}}-\mathbf{c}_{\mathrm{Si}}\right), & \mathbf{b}_{\mathrm{Si}}^{\prime}=1 / 2\left(\mathbf{b}_{\mathrm{Si}}-\mathbf{a}_{\mathrm{Si}}\right), & \mathbf{c}_{\mathrm{S} 1}^{\prime}=\mathbf{a}_{\mathrm{Si}}+\mathbf{b}_{\mathrm{S}_{1}}+\mathbf{c}_{\mathrm{S}_{1}}
\end{array}
$$

with $a_{\mathrm{Fe}}=2.866 \AA$ and $a_{\mathrm{Si}}=5.431 \AA$ at room temperature.

The epitaxy implies that the $a_{\mathrm{Fe}}^{\prime}$ parameter is strained to become equal to the $a_{\mathrm{S} 1}^{\prime}$ parameter inducing an in-plane strain equal to $\left(a_{\mathrm{Si}}^{\prime}-a_{\mathrm{Fe}}^{\prime}\right) / a_{\mathrm{S} 1}^{\prime}=0.06$. Consequently the $\mathrm{Fe}$ lattice expands by a factor $\gamma$ in the direction normal to the interface. The distance between two iron planes, parallel to the interface, is then $\gamma c_{\mathrm{Fe}}^{\prime} / 6$.

5.2 Fe-Si BOND. - Due to the ternary symmetry of the (111) Fe and Si layers, it is most probable that $\mathrm{Fe}$ atoms deposit on ternary $\mathrm{Si}$ adsorption sites. Three such sites are available: $\mathrm{T}_{4}$ and $\mathrm{H}_{3}$ sites (which derive their names from their geometrical location, respectively atop and hollow, and the number of chemical bonds, respectively four and three) and the $T$ site just above a silicon atom (Fig. 5).

In the $T_{4}$ position, the atom is bonded to three silicon atoms of the upper layer and to one of the subsurface, and is atop this one; in the $\mathrm{H}_{3}$ situation, the atom is in a hollow site and is bonded to three atoms of the surface layer. According to theoretical calculations [25], this site 


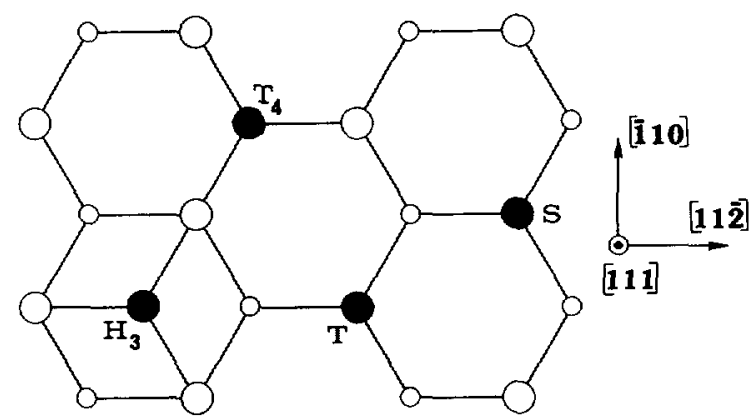

a)

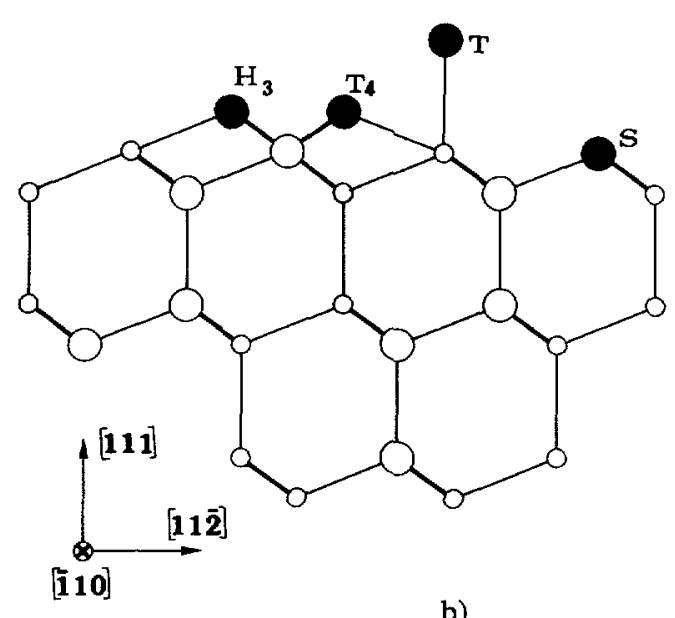

b)

Fig. 5. $-\mathrm{H}_{3}, \mathrm{~T}_{4}$ and $\mathrm{T}$ adsorption sites; $\mathrm{S}$ substitutional site. a) top view. b) side view.

is preferable for transition-metal atoms. The topmost site is the $T$ site, the highest site above the surface where the adsorbed atom has only one chemical bond with a silicon atom of the topmost layer and is just above it. This case could be a possibility for iron [22].

Another possibility is the substitution of the top silicon layer by adsorbate atoms, the socalled Missing Top Layer (MTL) model. This arises for arsenic deposited on Si (111) [26]. For Fe on Si (111) Urano et al. [23] proposed a two-step model : Fe atoms sit in 3-fold hollow sites on a silicon surface whose the top silicon plane is missing.

5.3 STACKING SEQUENCES OF Fe ATOMS. - The (111) atomic stacking in b.c.c. crystals can be described (Fig. 6) with a regular net of equilateral triangles. The atoms of the first layer are at the vertices of the triangles, those of the second one at the centre of one over two of the triangles and those of the third one at the centre of the other half of the triangles. This stacking is exactly the $\mathrm{ABCABC}$ stacking of the f.c.c. Iattice, the only difference is that the b.c.c. lattice is not compact. Once a layer is deposited which we shall call $\mathrm{A}$, then the succession of layers can be $B$ then $C$ or $C$ then $B$.

The hexagonal cell, defined in section 5.1, contains six atoms corresponding to six (111) layers. The coordinates of the Fe atoms of the three first layers are $(0,0,0),(2 / 3,1 / 3,1 / 6)$ and $(1 / 3,2 / 3,1 / 3)$ for one stacking (called here $\mathrm{ABC})$ and $(0,0,0),(1 / 3,2 / 3,1 / 6)$ and $(2 / 3,1 / 3$, 1/3) for the other stacking (ACB). 

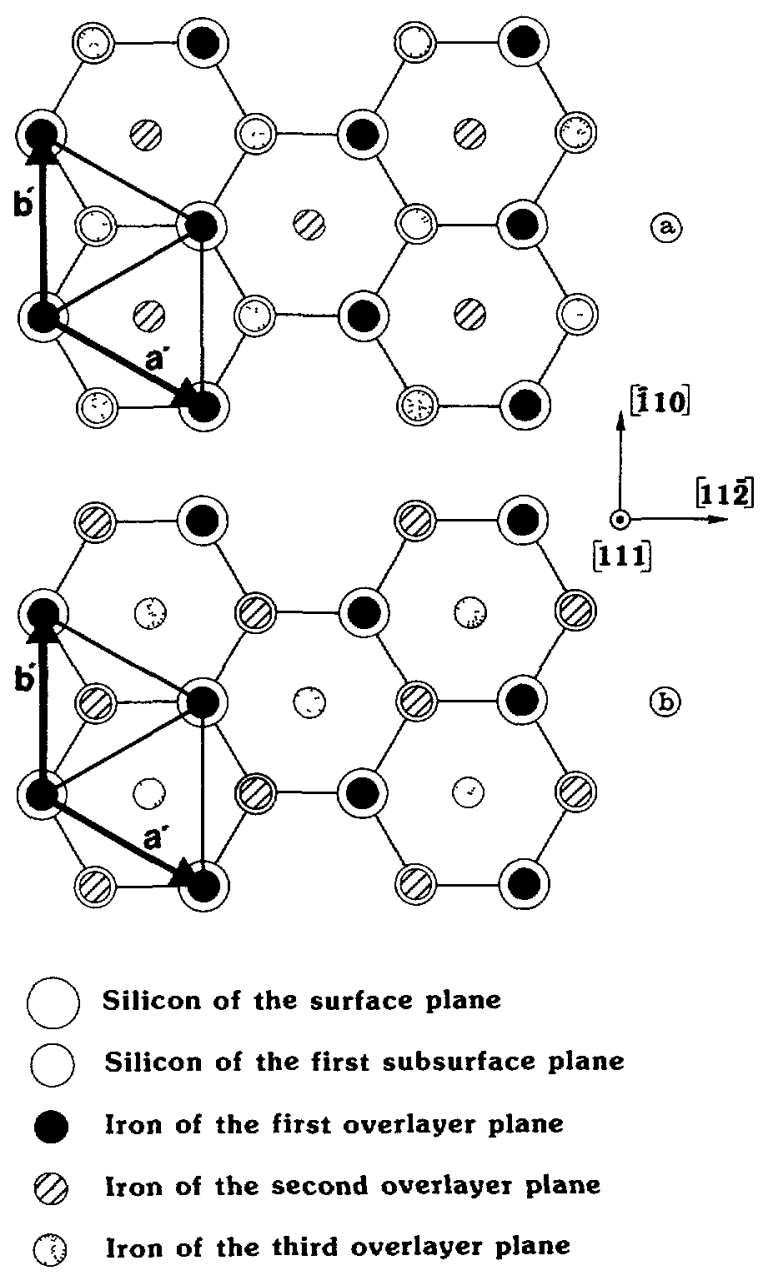

Fig. 6. - Stacking sequence projected along [111]. a) ABC. b) ACB.

These two stacking sequences, $\mathrm{ABC}$ and $\mathrm{ACB}$, can occur. They are deduced from one another by a $180^{\circ}$ rotation around the [111] cubic direction.

5.4 DisCUSSION. - The XSW experiments provide the parameters $F^{111}, P^{111}, F^{220}$ and $P^{220}$ and the unknowns are $N, d_{\mathrm{E}}^{111}, d_{\mathrm{E}}^{220}, d_{\mathrm{IF}}^{111}, f_{\mathrm{c}}^{111}$ and $f_{\mathrm{c}}^{220}$. If a pseudomorphic growth is assumed, the number of layers $N$ is determined from the R.B.S. data $(N=3$ here). $d_{\mathrm{E}}^{111}$ and $d_{\mathrm{E}}^{220}$, calculated in appendix II, depend on the dilatation factor $\gamma$. On the assumption that the strain normal to the interface is equal to the in-plane strain (Sect. 5.1), the $\gamma$ value is 1.06 and then $d_{\mathrm{E}}^{111}=0.877 \AA, d_{\mathrm{E}}^{220}=0.076 \AA$ for $\mathrm{ABC}$ stacking and $d_{\mathrm{E}}^{220}=$ $1.357 \AA$ for $\mathrm{ACB}$ stacking.

In the $\mathrm{ABC}$ stacking, $\mathrm{Fe}$ atoms are all very close to the 220 diffraction planes which is not the case for the ACB stacking (Appendix II and Fig. 7). An epilayer with a pure ACB structure would result in a fluorescence yield quite similar to the rocking curve (Fig. 8). This is in contradiction with the experimental curve (Fig. 4a).

If a pure $A B C$ stacking is assumed, the data (Tab. II and Fig. 9) lead to the site $\mathrm{T}$ for the 


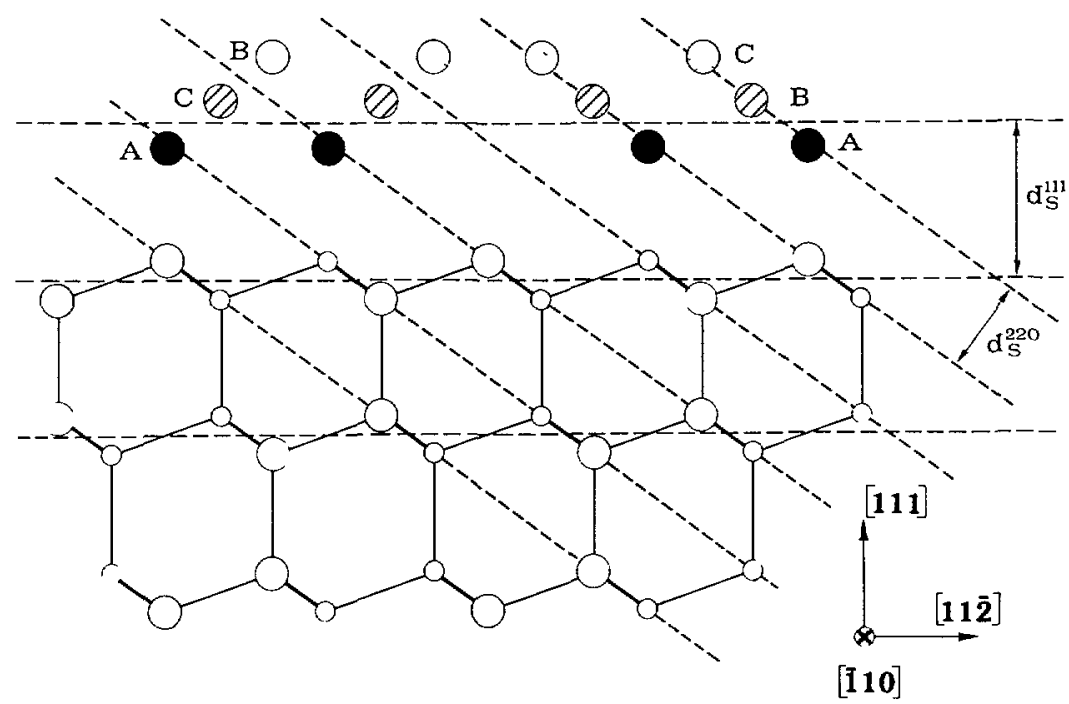

Fig. 7, - Side view of a silicon (111) surface covered with $3 \mathrm{ML}$ of $\mathrm{Fe}$ : $\mathrm{ABC}$ and $\mathrm{ACB}$ stacking sequences are shown.

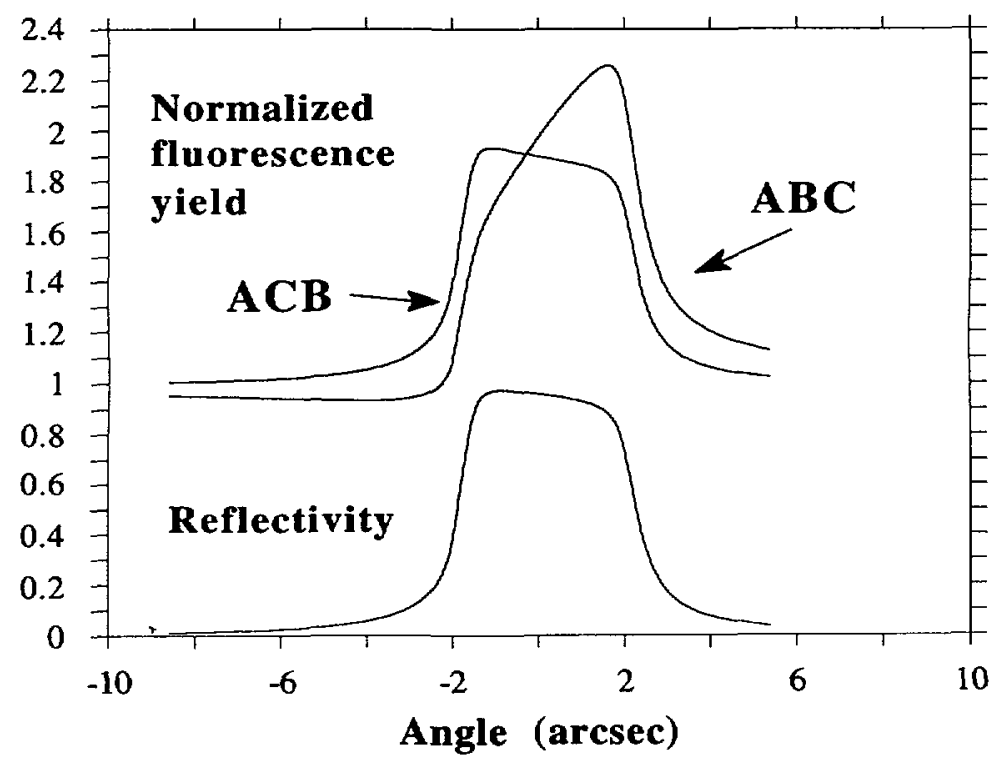

Fig. 8. - Theoretical rocking curve and fluorescence yields for 220 reflection and the two different stackings $\mathrm{ABC}$ and $\mathrm{ACB}(\lambda=1.2 \AA)$.

first Fe plane, and it so happens that the atoms of the second layer are just above $\mathrm{H}_{3}$ sites and those of the third layer above $\mathrm{T}_{4}$ sites. The $\mathrm{Si}-\mathrm{Fe}$ bond length is then $2.26 \pm 0.04 \AA$. The error bar on $d_{\mathrm{IF}}^{111}$ is much more larger than that on $d_{\mathrm{IF}}^{220}$. This is not surprising since $d_{\mathrm{E}}^{111}$ is roughly equal to a quarter of $d_{\mathrm{S}}^{111}$, and then for a fixed angular position on the rocking curve the $\mathrm{Fe}$ atoms are at different levels of the standing waves system. In bulk crystals such as $\mathrm{FeSi}, \mathrm{Fe}_{3} \mathrm{Si}, \alpha-\mathrm{FeSi}_{2}, \beta-\mathrm{FeSi}_{2}$ the Fe-Si bond length varies from $2.29 \AA$ to 


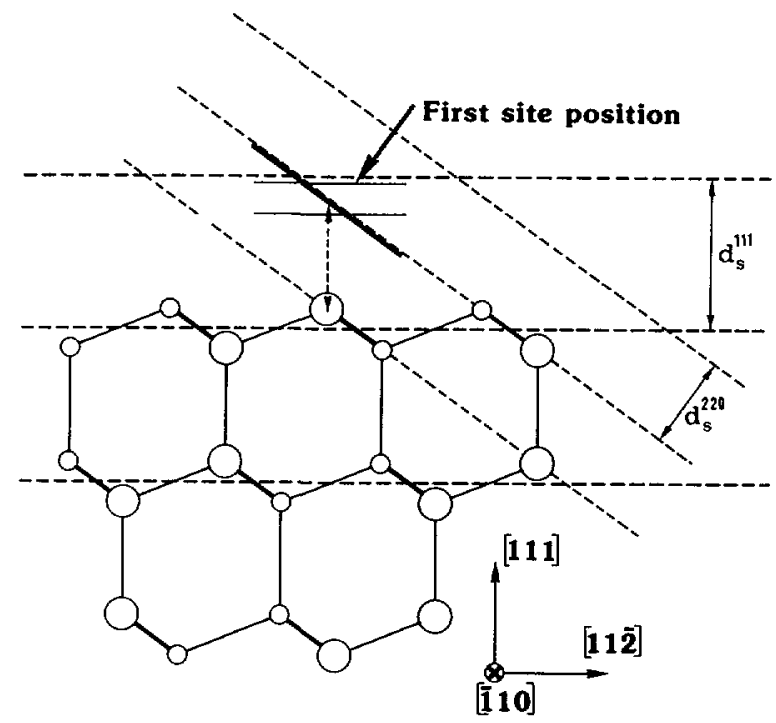

Fig. 9. - Fe-Si bond determined from $d_{\mathrm{IF}}^{111}$ and $d_{\mathrm{IF}}^{220}$. Note the large error bar for $d_{\mathrm{lF}}^{111}$.

$2.52 \AA$. It appears that the observed length is closed to the shortest value available in the literature. A surface relaxation (contraction) of the silicon surface has not been taken into account but it cannot be excluded.

The present work, which shows that the Fe atoms of the first layer occupy the $\mathrm{T}$ site, is in agreement with Urano et al. [22] but in contradiction with the $\mathrm{H}_{3}$ site predicted by theoretical calculations [25] (Tab. III). Let us mention that experimental studies by I-V LEED have had diverse interpretations : the on -top $T$ site is one of them [22] although the authors finally prefer 3-fold hollow sites on a MTL surface [23].

Table III. - Proposition for the first site of iron on a silicon (111) surface.

\begin{tabular}{|l|l|c|}
\hline \multicolumn{2}{|c|}{ Reference } & Proposal \\
\hline Theory & (Zeng-Ju 1987) & $\mathrm{H}_{3}$ \\
\hline I-V LEED & $\begin{array}{l}\text { (Urano 88) } \\
\text { (Urano 89) }\end{array}$ & $\begin{array}{c}\text { T } \\
\text { XSW }\end{array}$ \\
\hline
\end{tabular}

The coherent fractions $f_{\mathrm{c}}^{111}$ and $f_{\mathrm{c}}^{220}$ are equal to 0.24 (Tab. II), which is a low value. Three assumptions can be made to explain this result :

- a poor crystalline quality of the epilayer due to the low growth temperature ;

- a disorder induced by the amorphous silicon cap;

- a mixing of $\mathrm{ABC}$ and $\mathrm{ACB}$ domains.

The second hypothesis will be tested by studying, in a UHV system under construction, the same kind of deposit. 
In the case of an epilayer made with juxtaposed $\mathrm{ABC}$ and $\mathrm{ACB}$ domains, the coherent fraction, deduced from the 220 measurement, would be higher. However, the 111 reflection being insensitive to the mixing, $f_{c}^{111}$ can be taken as an upper limit (including the large error bar). Then numerical simulations show that the amount of ACB domains, if they are present, is lower than $20 \%$.

\section{Conclusion.}

The epitaxial growth of $\alpha$-Fe overlayer deposited at room temperature on silicon (111) substrate has been confirmed for a $3 \mathrm{ML}$ film using the $\mathrm{X}$-ray standing wave method. Two different stackings can occur and it has been shown that one of them is strongly predominant in the investigated sample. At the interface, iron atoms sit in $\mathrm{T}$ sites and the $\mathrm{Si}-\mathrm{Fe}$ bond length is equal to $2.26 \pm 0.04 \AA$. Iron atoms of the second layer are straight above $\mathrm{H}_{3}$ sites, and those of the third layer are above $\mathrm{T}_{4}$ sites.

To refine the structural model, further experiments are planned, using other reflections and also submonolayer deposits. An UHV system under construction will allow a comparison between films covered with amorphous silicon (this study) and as-grown epilayers.

\section{Acknowledgements.}

We thank the Laboratoire pour l'Utilisation du Rayonnement Electromagnétique (Orsay, France), where all experiments have been performed (beam line D15B, DCI storage ring). Thanks are due to Prof. Derrien of the Centre de Recherches sur les Mécanismes de Croissance Cristalline (Marseille, France) for the preparation of the sample. We thank F. Rocher of the Groupe de Physique des Solides (Univ. Paris VII \& Paris VI, CNRS, Paris, France) and M. G. Grimaldi of the Dip. Fisica of the Universita di Catania (Italy) for Rutherford Back Scattering calibrations. This research was supported by the European Communities through the ESPRIT Basic Research Action $n^{\circ} 3026$. The authors want to thank B. Gyors and M. Gaudet for their advice during the first steps of the computerization of the experiment and D. Joyeux of the Institut d'Optique (Orsay, France) for his help during the optical tests of the piezoelectric device.

\section{Appendix I.}

\section{Inclined symmetric reflections.}

I.1 $\rho_{\mathrm{h}}(\tau)$ : PROJECTED ATOMIC DISTRIBUTION ALONG THE DIFFRACTION VECTOR h. - Let $\mathrm{O} z$ be the axis normal to the crystal surface, $\mathrm{O} \tau$ the axis parallel to the diffraction vector $h$, $O \nu$ perpendicular to $\mathrm{O} \tau$ in the plane $(\mathrm{O} z, \mathrm{O} \tau)$ and $\mathrm{O} x$ the intersection of the plane $(\mathrm{O} z, \mathrm{O} \tau)$ with the crystal surface (Fig. 10). Let $\rho(x, z)$ be the projected atomic distribution on the $(\mathrm{O} x, \mathrm{O} z)$ plane which can be expressed as a function of the coordinates $\tau$ and $\nu, \rho^{\prime}(\nu, \tau)$, such that :

$$
\rho^{\prime}(\nu, \tau)=\rho(\tau \sin \phi+\nu \cos \phi, \tau \cos \phi-\nu \sin \phi) .
$$

It can be checked that when $\rho(x, z)$ is defined for $z=[0, t]$ and $x=[-\infty,+\infty]$, $\rho^{\prime}(\nu, \tau)$ is defined for $\nu=[\tau / \operatorname{tg} \phi-t / \sin \phi, \tau / \operatorname{tg} \phi]$ and $\tau=[-\infty,+\infty]$. The projected distribution $\rho_{\mathrm{h}}(\tau)$ is then :

$$
\begin{aligned}
\rho_{\mathrm{h}}(\tau)=\int_{\tau / \operatorname{tg} \phi-t / \sin \phi}^{\tau / \operatorname{tg} \phi} \rho^{\prime}(\nu, \tau) \mathrm{d} \nu & = \\
& =\int_{\tau / \operatorname{tg} \phi-t / \sin \phi}^{\tau / \operatorname{tg} \phi} \rho(\tau \sin \phi+\nu \cos \phi, \tau \cos \phi-\nu \sin \phi) \mathrm{d} \nu .
\end{aligned}
$$




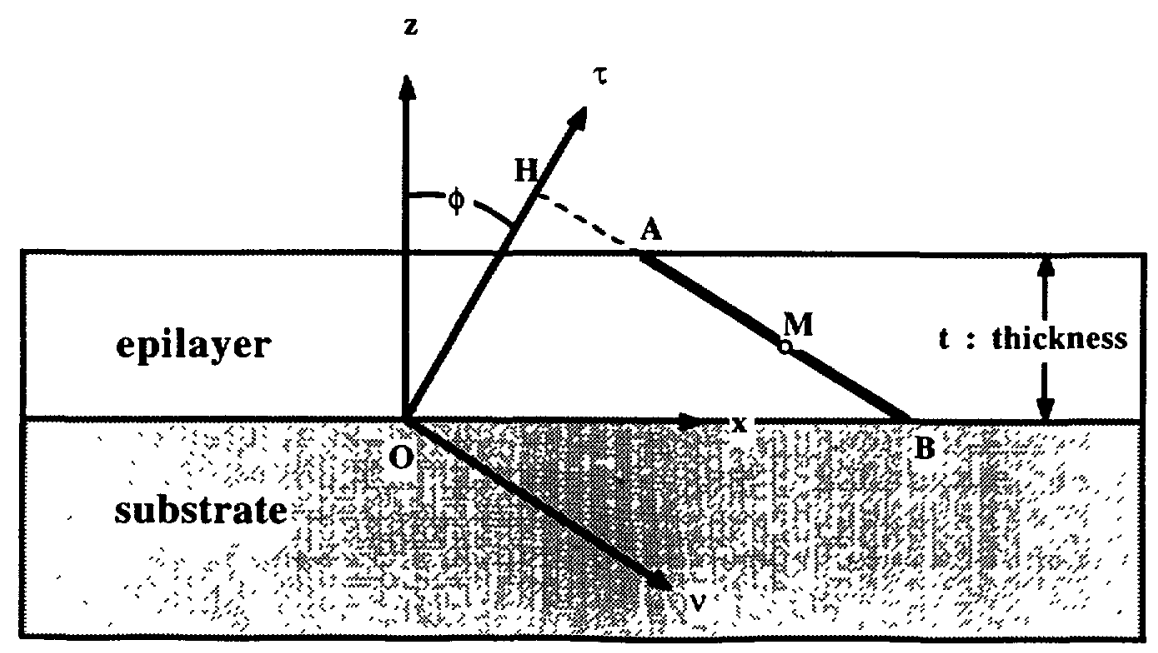

Fig. 10. - System of coordinates used in the appendix I. For a given value of $\tau, \nu=\overline{\mathrm{HM}}$ varies from $\overline{\mathrm{HA}}=\tau / \operatorname{tg} \phi-t / \sin \phi$ to $\overline{\mathrm{HB}}=\tau / \operatorname{tg} \phi$. The coordinate $\nu^{\prime}$ is equal to $\overline{\mathrm{AM}}$.

When replacing $\nu$ by $\nu^{\prime}=\nu-\tau / \operatorname{tg} \phi+t / \sin \phi$, one obtains :

$$
\rho_{\mathrm{h}}(\tau)=\int_{0}^{t / \sin \phi} \rho\left(\tau / \sin \phi+\nu^{\prime} \cos \phi-t / \operatorname{tg} \phi,-\nu^{\prime} / \sin \phi+t\right) \mathrm{d} \nu^{\prime}
$$

I. $F$ AND $P$ PARAMETERS IN THE EXPITAXIAL CASE. - In the epitaxial case, the epilayer and the substrate have commensurate in-plane periods. If one considers the most simple case where the epilayer and substrate have the same period $a_{x}$ along the $\mathrm{O} x$ direction, then :

$$
\rho(x, z)=\rho\left(x+m a_{x}, z\right)
$$

where $m$ is an integer. From the expression of $\rho_{\mathrm{h}}(\tau)$, it can be seen that $\rho_{\mathrm{h}}(\tau)$ is also a periodic function whose period $\tau_{\mathrm{h}}$ is equal to $a_{x} \sin \phi$, since :

$\rho_{\mathrm{h}}\left(\tau+m a_{\imath} \sin \phi\right)=$

$$
=\int_{0}^{t / \sin \phi} \rho\left(\tau / \sin \phi+m a_{x}+\nu^{\prime} \cos \phi-t / \operatorname{tg} \phi,-\nu^{\prime} / \sin \phi+t\right) \mathrm{d} \nu^{\prime}=\rho_{\mathrm{h}}(\tau) .
$$

Furthermore, for inclined symmetric reflections $h k \ell$, it can be easily checked that this period $\tau_{\mathrm{h}}$, equal to $a_{x} \sin \phi$, is a multiple of $d_{k k l}$ :

$$
\tau_{\mathrm{h}}=a_{x} \sin \phi=n d_{h k \ell} .
$$

Thus $F$ and $P$ have non zero values by renormalizing the integrals:

$$
F \mathrm{e}^{l 2 \pi P}=\lim _{m \rightarrow+\infty} \frac{\int_{-m \tau_{\mathrm{h}}}^{m \tau_{\mathrm{h}}} \rho_{\mathrm{h}}(\tau) \mathrm{e}^{t 2 \pi \tau / d_{h k \ell}} \mathrm{d} \tau}{\int_{-m \tau_{\mathrm{h}}}^{m \tau_{\mathrm{h}}} \rho_{\mathrm{h}}(\tau) \mathrm{d} \tau}=\frac{\int_{\text {over } \tau_{\mathrm{h}}} \rho_{\mathrm{h}}(\tau) \mathrm{e}^{\mathrm{r} 2 \pi \tau / d_{h l \ell}} \mathrm{d} \tau}{\int_{\text {over } \tau_{\mathrm{h}}} \rho_{\mathrm{h}}(\tau) \mathrm{d} \tau}
$$




\section{Appendix II.}

Fe position with respect to the silicon diffraction planes.

Let us call $u, v, w$ the coordinates of one Fe atom in the undeformed hexagonal Fe cell. After distortion due to epitaxy, these coordinates expressed in the $\mathrm{Si}$ hexagonal cell become $\left(u / 2, v / 2, \gamma\left(a_{\mathrm{Fe}} / a_{\mathrm{S} 1}\right) w\right)$. The three layers are described for the two orientations by the $u, v, w$ coordinates given in section 5.3 where now $u$ and $v$ are given modulo an integer.

Let us call $r_{h k l}$ the projection of the position vector $\mathbf{r}$ of a $\mathrm{Fe}$ atom on the normal to the $h k \mathcal{l}$ diffraction planes of silicon expressed in unit equal to the $h k \mathcal{l}$ diffraction planes distance $d_{S}^{h k \ell}$. The origin for $\mathbf{r}$ is taken on a Fe atom of the first layer (A atom).

Then

$$
\begin{aligned}
& r_{h k \ell}=(h-\ell) u / 2+(k-h) v / 2+\gamma\left(a_{\mathrm{Fe}} / a_{\mathrm{S}_{1}}\right)(h+k+\ell) w \text { leading to : } \\
& r_{111}=3 \gamma\left(a_{\mathrm{Fe}} / a_{\mathrm{S} 1}\right) w \\
& r_{220}=u+4 \gamma\left(a_{\mathrm{Fe}} / a_{\mathrm{S}_{1}}\right) w .
\end{aligned}
$$

The first result $r_{111}$ is quite obvious and naturally does not depend on the stacking. It induces a $d_{\mathrm{E}}^{11} / d_{\mathrm{S}}^{111}$ value equal to $\gamma\left(a_{\mathrm{Fe}} / a_{\mathrm{Si}}\right)$ whose value is 0.2797 , with $\gamma=1.06$. The value $r_{220}$ depends on the stacking. For $\mathrm{ABC}$ stacking the $r_{220}$ values for sites $\mathrm{B}$ and $\mathrm{C}$ are respectively 0.0396 and 0.0792 modulo an integer, giving a $d_{\mathrm{E}}^{220} / d_{\mathrm{S}}^{220}$ value equal to 0.0396 , whereas for ACB stacking the corresponding values are 0.7069 and 0.4125 modulo an integer leading to $d_{\mathrm{E}}^{220} / d_{\mathrm{S}}^{220}$ equal to 0.7069 .

\section{References}

[1] Bost M. C., Mahan J. E., J. Appl. Phys. 58 (1985) 2696.

[2] Lefki K., Muret P., Cherief N., Bustarret E., Nguyen T., Boutarek N., Madar R., Chevrier J., Derrien J., Brunel M., Solid State Commun. 80 (1991) 791.

[3] Derrien J., Chevrier J., Younsi A., Le Thanh V., Dussaulcy J. P., Cherief N., to be published in Physica Scripta.

[4] Mahan J. E., Geil K., Robinson G. Y., Long R. G., Xinghna Y., Bai G., Nicolet M. A., Mathan M., to be published in Appl. Phys. Lett.

[5] Kanaji T., Urano R., Hiraki A., Iwani M., supp. to « Le Vide, les Couches Minces 201 (1980) 117.

[6] Vlieg E., Fontes E., Patel J. R., Phys. Rev. B 43 (1991) 7185.

[7] Zegenhagen J., Patel J. R., Freeland P. E., Tong R. T., Phys. Rev. B 44 (1991) 13626.

[8] Batterman B. W., Phys. Rev. 133 (1964) A 759.

[9] Batterman B. W., Phys. Rev. Lett. 22 (1969) 703.

[10] Authier A., Acta Cryst. A 42 (1986) 414.

[11] Golovchenko J. A., Batterman B., Brown W. L., Phys. Rev. B 10 (1974) 4239.

[12] Andersen S. K., Golovchenko J. A., MaIr G., Phys. Rev. Lett. 37(1976) 1141.

[13] Vlieg E., Fischer A.E.M.J., Van der Veen J. F., Dev B. N., Materlick G., Surf. Sci. 178 (1986) 36.

[14] Saitoh Y., Hashizume H., Tsutsui K., Jpn J. Appl. Phys. 27 (1988) 1386. 
[15] Capelle B., Pétroff J. F., Boulliard J. C., Lifchitz A., Malgrange C., Ferret D., BERNARD Y., Frouin J., A new experimental XRSW set up at LURE. Conf. Proceedings 25, "Second European Conference on Progress in X-ray Synchrotron Radiation Research" (Rome, 2-6 October 1989) A. Baberna, E. Bernieri and S. Mobilio Eds. (SIF, Bologna, 1990).

[16] FERRET D., Thèse de doctorat de l'Université Paris VI (1990).

[17] JACKsON J. D., Classical Electrodynamics (New York, 1975), p. 848.

[18] Du MOND J. W., Phys. Rev. 52 (1937) 872.

[19] BONSE U., HART M., Appl. Phys. Lett. 7 (1965) 238.

[20] Kikuta S., Kohra K., J. Phys. Soc. Jpn 29 (1970) 1322.

[21] Hashizume H., Sauvage M., Pétroff J. F., Riglet P., Capelle B., Rapport d'activité, Lure (1979).

[22] Urano T., Kanaji T., Appl. Surf. Sci. 33/34 (1988) 68.

[23] Urano T., Kaburagi M., Hongo S., Kanaji T., Appl. Surf. Sci. 41-42 (1989) 103.

[24] Gavriluuk Y. L., Kachanova L. Y., Lifshits V. G., Surf. Sci. Lett. 256 (1991) L589.

[25] Zeng-Ju T., Satoko C., Onishi S., Phys. Rev. B 36 (1987) 6390.

[26] Patel J. R., Golovchenko J. A., Freeland P. E., Gossmann H. J., Phys. Rev. B 36 (1987) 7715. 